\title{
AIE Stereoisomers with Huge Differences in Luminescence Behavior and Biomedical Activity
}

\author{
Xuewen He, ${ }^{\dagger, \ddagger}$ Ryan T. K. Kwok, ${ }^{\dagger, \ddagger}$ Jacky W. Y. Lam, ${ }^{\dagger, \ddagger}$ Ben Zhong Tang ${ }^{*,+, \ddagger, \square}$ \\ ${ }^{\dagger}$ Department of Chemistry, The Hong Kong Branch of Chinese National Engineering Research Centre \\ for Tissue Restoration and Reconstruction, Institute for Advanced Study, Department of Chemical and \\ Biological Engineering, Division of Life Science and State Key Laboratory of Molecular Neuroscience, \\ The Hong Kong University of Science and Technology, Clear Water Bay, Kowloon, Hong Kong, China \\ ${ }^{\ddagger}$ HKUST-Shenzhen Research Institute, Shenzhen, 518057, China \\ ${ }^{\square}$ Center for Aggregation-Induced Emission, SCUT-HKUST Joint Research Laboratory, State Key \\ Laboratory of Luminescent Materials and Devices, South China University of Technology, Guangzhou, \\ 510640, China
}

*B.Z.T: tangbenz@ust.hk

ABSTRACT: Stereoisomers that differ only in spatial orientation of their atoms could exhibit distinctive properties and have attracted immense interest in drug development and material science. Herein, a series of AIE-featured stereoisomers with pronounced difference in luminescent and biomedical activities were efficiently synthesized and easily purified. One of the isomers in the E/Z pair is emissive with high fluorescence quantum yield at room temperature, while the other one is nearly non-emissive. As the isomers could transform between each other under UV irradiation, the light-up cell imaging was successfully demonstrated using the non-emissive isomer as a turn-on probe via isomerization process. Analysis of crystal packing patterns illustrated that the contrasts in void space possibly caused the difference in molecular motion and thus led to their distinct luminescence properties. Further, the E/Z isomers displayed remarkable difference in enzymatic conversion rates and cellular toxicities for several cancer cell lines. The distinctive luminescence properties of the isomeric pair provided a powerful tool to image those divergencies in biomedical activity, holding great potential for visualizable drug screening and development.

\section{INTRODUCTION}

Exploring the relationship between molecular structure and property is crucial to the development of material science. Isomers with the same composition but differing in constitution, stereochemistry and chirality usually exert huge different effects on molecular engineering and life science. Although many efforts have been devoted to investigating the dramatic role of molecular chirality in dictating their optical properties and drug potency, much less work has been done to learn how the $E / Z$ stereoisomers could modulate their properties. A few cases of geometric $E / Z$ isomers have been reported to differ in their drug properties. ${ }^{1,2}$ For 
instance, Z-tamoxifen is a weak estrogen agonist, but its $E$-cousin behaves as an effective estrogen antagonist in treating breast cancer. ${ }^{3,4}$ Cis and trans-platin show distinct activities toward the target, in which cisplatin exhibited high potency to various types of cancer, ${ }^{5,6}$ while the trans-platin shows negligible anticancer activity. ${ }^{7}$ Recently, some fluorescent stereoisomer probes were reported with distinct binding affinities to enzyme or DNA target. ${ }^{8,9}$ However, there is still a big challenge to track stereoisomers in the process of cellular endocytosis, biotarget binding and responsive conversion, and finally to visualize their distinctive biomedical activities.

The efficient synthesis of $E / Z$ stereoisomers with distinct luminescence properties is important to elucidate their individual characteristics, interactions and may offer a unique opportunity to explore their practical applications for molecular engineering and drug discovery. The stereochemistry of carbon-carbon double bonds of aggregation-induced emission luminogens (AIEgens), such as the triphenylethylene, tetraphenylethylene and their derivatives are well-suited for such studies because their $E / Z$ configurations can be readily prepared by the McMurry coupling of benzophenone carrying asymmetric functional groups. ${ }^{10}$ Additionally, they exhibit AIE properties with efficient luminescence emission in aggregate state via the restriction of intramolecular motion, featuring large absorptivity, robust luminosity and strong photobleaching resistance. ${ }^{11}$ These exceptional luminescence properties overcome the problems of aggregation caused quenching and photobleaching from traditional fluorophores $^{12,13}$ and enables a wide range of practical applications based on functionalized AIEgens for photoelectronic devices, ${ }^{14-16}$ chemical/bioanalyte sensing, ${ }^{17-23}$ molecular imaging, ${ }^{24-26}$ dynamic process tracking ${ }^{27,28}$ and photo responsive therapeutic applications. ${ }^{29-33}$

However, due to the difficulties in separation caused by little difference in polarity, the study of AIE-featured pure isomers is still in the infancy. Although palladium-catalyzed coupling reactions were attempted to directly synthesize pure isomers, ${ }^{34}$ it was found that the expected $Z$ isomer is usually accompanied with $E$ isomer as a minor product. ${ }^{35,36}$ Strategies such as attaching bulky groups to the AIE core or stabilizing it with multiple hydrogen bonds to enlarge size and improve polarity differences had successfully realized the separation of some AIE stereoisomers. $^{37,38}$ Nevertheless, the complexities in molecular design would limit their practical use. Consequently, mixtures of the $E / Z$ isomers were prepared and used in most applications, ${ }^{39-41}$ leading to compromised effects on the properties and functions of these molecules. Another tough task is to identify $E / Z$ geometry after separation. Current approach relies on growing single crystals for X-ray diffraction to confirm the isomeric geometry, ${ }^{42-44} \mathrm{a}$ tedious task and only applicable to those isomers that can easily pack into single crystals. 
Although a few separated isomers possess different optical property, ${ }^{37,38,45-47}$ such as emission wavelength shifts and fluctuation in fluorescent brightness, it is still very difficult to precisely discriminate individual isomeric type, let alone to monitor their potential distinct biomedical activities. As the isomerization is closely associated with the molecular motion, the stereoisomer is often unstable in solution and causes changes in luminescence properties. ${ }^{45}$ Therefore, it is highly desirable to obtain stereoisomers that possess simple structures, high stabilities that could be engineered to robust forms, and trackable characteristics for the study of their properties and related applications.

Herein, a series of AIE stereoisomers with simple molecular structures were efficiently synthesized and easily separated (Scheme 1). The $E / Z$ isomer in each pair exhibited huge difference in luminescence properties and biomedical activities. That is, one isomer in the $E / Z$ pair is emissive with a high quantum yield, while the other one is non-emissive in the solidstate. The divergence in luminescence properties of the precursor could be inherited by the derivatives with keeping the stereo configuration without any extra separation. The emissive and non-emissive characters could be applied for the precise discrimination of the $E / Z$ geometric structures. Due to their capabilities of isomerization, the application of the nonemissive isomer for light-up bioimaging under photoirradiation was successfully demonstrated. Further, the $E / Z$ isomers displayed different enzymatic conversion rates and distinct cytotoxicity toward a variety of cancer cell lines, holding a great potential in pharmaceutical candidate development and screening.

\section{RESULTS AND DISCUSSION}

\section{Synthesis, Separation and Characterization of Luminescent Properties}

The starting stereoisomers, including E/Z-TPAN-M, E/Z-TPAN-OM, E/Z-TPAN-NMBr and E/Z-TPAN-NM were easily synthesized through one-step reaction (scheme 1) To obtain pure stereoisomer for investigation of its properties, separation of isomers was first tried for $E / Z$ TPAN-M mixture with two distinctive nucleic magnetic resonance (NMR) peaks for the methyl proton (2.41 ppm and $2.28 \mathrm{ppm}$ ). Unfortunately, the common C18 reverse column was unable to realize their separation as they always eluted out at the same time, no matter what ratio of elution solvents was employed in high performance liquid chromatography (HPLC). After incorporating an electron donor group, methoxy, isomer pairs E/Z-TPAN-OM could be easily separated because of apparent difference in elution time. Compared to the previously reported isomers that need to stretch their molecular structure to a pretty large degree for separation, the E/Z-TPAN-OM isomers with simple structures would benefit from their easy and efficient 
synthesis and versatile structural modifications. The purified isomers of E/Z-TPAN-OM were confirmed by ${ }^{1} \mathrm{H}$ and ${ }^{13} \mathrm{C}$ NMR spectra, high-resolution mass spectra as well as X-ray single crystal diffraction. Noteworthy, in the ${ }^{1} \mathrm{H}$ NMR spectra of $Z$ and $E$-TPAN-OM, the protons resonance in the methyl group appeared at $3.86 \mathrm{ppm}$ and $3.77 \mathrm{ppm}$, respectively; and the aromatic protons also showed apparent differences with peaks at 7.02-7.00 ppm (zone 1) and 6.71-6.68 ppm (zone 2), that belong to the $Z$ and $E$ isomer, respectively. These remarkable differences could be employed for their assignments and monitoring their structural evolutions. The derivatives including E/Z-TPAN-OH, E/Z-TPAN-POE and E/Z-TPAN-POH perfectly inherited the stereo configurations as they could be synthesized under very mild conditions. As a result, no further separation was needed. Their purities and geometries were also confirmed by the same measurements as described above.

The successful purifications of these isomers make it feasible to study their individual optical properties. In the solution state, E/Z-TPAN-POH isomers shared similar absorption spectra in the ultraviolet band with maximums at $332 \mathrm{~nm}$. Surprisingly, their AIE curve in DMSO/water showed huge difference with 0.5 -fold and 30 -fold enhancement in emission intensity for ZTPAN-OM and E-TPAN-OM, respectively, at 99.5\% water fraction (Figure 1). TEM images clearly showed their differences in aggregation morphology, in which E-TPAN-OM inclined to form regular rod-like shapes and even arranged into a two-dimension plane at $99.5 \%$ water fraction. In contrast, Z-TPAN-OM could not form regular morphologies. Encouraged by those remarkable difference in the solution state, the properties of the stereoisomers in solid-state were next investigated. As shown in Figure 2a, both of E/Z-TPAN-OM isomers showed redshifted absorption spectra compared to the ones in solution state, indicating the formation of much higher planar conformations. Additionally, larger red-shift occurred for the $E$ isomer, possibly due to its relatively stronger donor-acceptor (D-A) structure. Regarding its fluorescence properties, once again there was a significant difference in fluorescence brightness, in which $E$-TPAN-OM was ultrabright with quantum yield up to $21.8 \%$, while the $Z$ isomer was nearly dark (Figure $2 \mathrm{~b}, \mathrm{c}$ ). The $E$ isomer had a fluorescence lifetime of $938 \mathrm{ps,}$ which was 4 times longer than its $Z$ counterpart, further demonstrating the much higher efficiency of the radiative transition in E-TPAN-OM. When E/Z-TPAN-OM were cooled down to cryogenic temperature (CT, $77 \mathrm{~K})$, both of them emitted strong fluorescence but with green and blue color, respectively. The significant enhancement in fluorescence intensity at CT could be attributed to the higher degree in restriction of intramolecular motions, confirming that both of them were typical AIE-featured luminogens (Figure 2c). The powders of both E/Z-TPANOM were identified as crystalline morphology, as sharp peaks appeared in their XRD patterns. 
The fluorescence spectra were also measured under temperature evolution. As shown in Figure $2 \mathrm{~d}$, the emission intensity of Z-TPAN-OM swiftly decreased to nearly 0 when the temperature rose up to $160 \mathrm{~K}$. On the contrary, the $E$ isomer displayed much slower rate in decrease of emission intensity and could preserve about $20 \%$ even when approaching the room temperature. The dynamic change of fluorescence from $77 \mathrm{~K}$ to RT of $E$ and $Z$-TPAN-OM with blue and green color fluorescence emission, respectively, was also real-time captured in the Video 1.

\section{E/Z Isomerization and Light-up Bioimaging}

The isomerization was studied next in E/Z-TPAN-OM. As the protons in the methyl group and aromatic zone showed distinct chemical shifts, NMR spectroscopy was first employed to monitor the isomerization process. Quartz tubes filled with $\mathrm{CDCl}_{3}$ solutions of $E / Z$-TPAN-OM were placed under $365 \mathrm{~nm}$ UV irradiation at room temperature. As shown in Figure 3a, the resonances of the characteristic protons of E-TPAN-OM appeared in the Z-TPAN-OM samples (denoted as zone 4 for methyl group and zone 3 for aromatic zone) after irradiation, demonstrating its capability to transform to its isomeric counterpart. Similarly, the transformation of $E$-TPAN-OM to its $Z$ counterpart was also realized under UV irradiation in which the characteristic proton resonances of the $Z$ isomer in zone 2 and 4 appeared. The irradiation time dependent transformation processes for $E$ and Z-TPAN-OM were depicted by proton NMR resonance. In contrast, samples stored under ambient light showed negligible change in NMR spectra even after 7 days, suggesting their stability in solution state. Due to the different elution times, the isomerization process could also be monitored via HPLC. As shown in Figure 3b, the appearance of peaks in zone 5 and zone 6 after UV irradiation could be assigned to the freshly generated $Z$ and $E$ isomer, respectively, as they located in the same elution position as the original isomer. Prolonged irradiation time led to a dramatic decrease in the peak intensity of the $E$ isomer accompanied with increase in the ones of $Z$ isomer and vice versa. The ratio of $Z$ to $E$ isomer was about 1.14 after $150 \mathrm{~min}$ of irradiation and close to the balance. In addition, the fresh peak with longer elution time could be assigned to the photocyclized side-product with less polarity as indicated by the mass spectra. This process was similar to the previously reported photocyclization that usually happened in the aromatic ring constituted AIE systems. ${ }^{48}$

As they are capable of isomerizing into each other, the non-emissive Z-TPAN-OM was expected to be applicable in the light-up bioimaging by transforming to its emissive $E$ counterpart. As shown in Figure 4, no apparent aggregation occurred outside the cellular membrane after incubation with the $Z$ isomer. The fluorescence signal could neither be detected 
under microscopy because of the inherent non-emissive property of Z-TPAN-OM. Surprisingly, after 2 min of irradiation under the mercury lamp, the HeLa cells lit-up with scattered greencolor fluorescence signal. The blue-color fluorescence was probably originated from the photocyclization of Z-TPAN-OM accompanying the isomerization process. No signal appeared in the control cells without incubation with Z-TPAN-OM, indicating the light-up fluorescence signal indeed originated from the isomerization process. On the other hand, when E-TPAN-OM was incubated with living cell, rod-like aggregates formed rapidly in the medium, and it seemed hard to enter the cell, with green-color fluorescence only came from the extracellular membrane. This rapid aggregation manner of E-TPAN-OM agreed well with the phenomenon recorded by TEM image. The dynamic process of light-up cell imaging using Z-TPAN-OM was investigated next. The intensity of the green-color fluorescence reached plateau in just 2 min, indicating rapid responsiveness in the living cell. Furthermore, the light-up imaging could be operated with precise spatial control, with fluorescence signal generated only in the area under irradiation. Complementary to the previous reported photocyclization-activated turn-on fluorescence, the photoisomerization process provides another potential strategy to realize light-up bioimaging.

\section{Mechanism Study for the Divergent Luminescent Properties}

Void space in the molecular crystal packing is able to provide the free space for molecular motion to exert influence toward luminescence behavior. ${ }^{49} \mathrm{X}$-ray diffraction analysis revealed that the single crystals of $E$ and Z-TPAN-OM were in the P21/n and P21/c space groups, respectively (Figure 5a,b). For E-TPAN-OM, each molecule was connected to 6 nearby molecules with 10 weak intermolecular interactions. Cavities could be observed in the threedimensional packing structure of $E$-TPAN-OM with pores along the $c$ axis at room temperature (Figure 5c). Meanwhile, for the Z-TPAN-OM, each of the molecule linked with 3 nearby molecules through 6 weak intermolecular interactions. The pores in the packing structure became much conspicuous along the $c$ axis (Figure 5d). The calculated void space in crystal packing of Z-TPAN-OM was up to $3.311 \AA^{3}$ at $160 \mathrm{~K}$, nearly 8-fold bigger than its $E$ counterpart at the same temperature (as $V_{\mathrm{V}, \mathrm{Z}} / V_{\mathrm{V}, \mathrm{E}}$ in Figure 5e). Throughout the tested temperature range from $100 \mathrm{~K}$ to $300 \mathrm{~K}$, the void space of $Z$ isomer continued to be much larger in comparison. It is expected that the larger free space in $Z$ isomers would possibly allow their intramolecular motion leading to a reduction in the emission efficiency. On the other hand, the much smaller void space in the $E$ isomers would be able to constrain molecular motion resulting in the observed robust emission. 
Another set of isomers, E/Z-TPAN-OH, presented similar divergency in their fluorescence properties. The AIE emission intensities enhanced by 1.92-fold and 11.27-fold for $E$ and $Z$ isomer at $99.8 \%$ water fraction, respectively, showing their different AIE properties (Figure 6a). Huge difference in emission intensity could also be observed in the solid-state with a quantum yield of $13.72 \%$ for Z-TPAN-OH but not detectable (ND) for the $E$ isomer (Figure $6 \mathrm{~b}$ ). The isomerization between $Z$ and $E$-TPAN-OH under UV light irradiation were also verified by ${ }^{1} \mathrm{H}$ NMR spectra and HPLC elution. The cell imaging test showed that neither of them could be internalized into the living cell, as they both rapidly aggregated into macrostructures. However, the emissive and non-emissive characters were maintained for the $Z / E$ isomer, respectively. The crystal packing patterns of E/Z-TPAN-OH presented obvious cavities (Figure 6c,d). But the volume of void space displayed drastic difference, in which the values from $E$-TPAN-OH was always larger than the $\mathrm{Z}$ isomer, and the values of $V_{\mathrm{V}, \mathrm{E}} / V_{\mathrm{v}, \mathrm{Z}}$ in all tested temperature surpassed 17 (Figure 6e). Guided by the same principle for E/Z-TPAN-OM pair, the lower degree in crystal void volume in Z-TPAN-OH afforded its higher efficiency in fluorescence when compared to $E$-TPAN-OH. The configuration reversal from $E$-TPAN-OM to Z-TPAN-OH that showing much stronger fluorescence compared to their individual isomeric counterpart, indicated the significant role of aggregated pattern in shaping the material properties apart from effects from molecular structure. Theoretical calculation for the HOMO and LUMO energy levels were further conducted in E/Z-TPAN-OM and E/Z-TPAN-OH pairs. Compared to their individual non-emissive counterpart, both E-TPAN-OM and Z-TPAN-OH possessed lower reorganization energy in $100 \mathrm{~K}$. These differences became much bigger when the temperature increased to room temperature, suggesting the sharp contrast in fluorescence efficiency mainly came from their distinct non-radiative transition. Extending to the case of E/Z-TPAN-POE, the brighter $\mathrm{Z}$ isomer still displayed smaller void space in its crystal pattern, indicating the positive correlation between aggregated pattern and fluorescence emission efficiency in those fluorophores. It was observed that the Z-type isomers, including Z-TPAN-POH, Z-TPAN$\mathrm{NMBr}$ and Z-TPAN-NM, all showed remarkable higher fluorescence quantum yield than their individual $E$ counterparts, possibly following the same relationship between packing pattern and luminescence properties.

\section{Divergency in Enzymatic Responsiveness}

As the cellular uptake of E/Z-TPAN-OM and E/Z-TPAN-OH was limited, E/Z-TPAN-POH modified with phosphate group were further designed to improve their water solubilities and endow them with enzymatic responsiveness, as the phosphate group is the substrate of alkaline 
phosphatase (ALP) that plays a significant role in cell signaling pathway and has become an important biomarker in cancer diagnosis. ${ }^{50-53}$ The obtained E/Z-TPAN-OM isomers retained the same stereo-structure as their individual precursor and displayed different fluorescence quantum yields. As the E/Z-TPAN-POH and their hydrolysis product, E/Z-TPAN-OH (Figure 7a), eluted out with different time scales, the ALP-catalyzed hydrolysis of E/Z-TPAN-POH could therefore be quantitatively monitored by HPLC. As shown in Figure $7 \mathrm{~b}$, as the reaction proceeded, the intensity of the peak of Z-TPAN-POH continuously decreased, accompanied by increased peak intensity of the product with the same elution time as Z-TPAN-OH. Similarly, the hydrolysis of E-TPAN-POH also led to apparent decrease of its peak intensity and increase in the production of E-TPAN-OH. Importantly, nearly 4.2-fold faster hydrolysis rate was observed for E-TPAN-POH than its $Z$ counterpart, indicating that the nuance in structure could leverage big difference in their enzymatic recognition and reaction (Figure 7c). To gain insights into the binding mode of E/Z-TPAN-POH with ALP, we carried out molecular-docking studies with the structure of ALP. More than three times lower binding energy proved that the binding affinity of ALP with $E$-TPAN-POH at its active site was much stronger than the $\mathrm{Z}$ counterpart. This striking divergency in enzymatic responsiveness to stereoisomers is similar to known drugs, e.g. cisplatin that displays remarkable activity towards the target compared to its trans counterpart. ${ }^{6}$

\section{Diversified Cellular Toxicity}

Inspired by the difference of E/Z-TPAN-POH in ALP responsiveness, their intracellular toxicities were further investigated as ALP expression level was commonly upregulated in cancer cells caused by dysfunctional biochemical and metabolic processes. ${ }^{54,55}$ As shown in Figure 8, after incubation with HeLa cells for 2 hours, the E/Z-TPAN-POH exhibited distinct toxicities. The cell survival rate rapidly decreased to 0 for Z-TPAN-POH when the concentration reached $300 \mu \mathrm{M}$. In sharp contrast, the cell viability retained nearly $100 \%$ for the $E$ isomer. This cellular toxicity was also dependent on incubation time. When the time extended to $12 \mathrm{~h}$ the toxicity of the $Z$ isomer became more pronounced, as $200 \mu \mathrm{M}$ isomer could completely kill the cells. The cell viability for $E$ isomer, however, maintained above $70 \%$ at a concentration of $800 \mu \mathrm{M}$. The sharp contrast of toxicities was also evidenced by the destroyed cellular morphologies after incubation with Z-TPAN-POH and the intact morphologies with its $E$ counterpart, undoubtedly verified their dramatical differences in cell toxicity. This correlation between toxicity and $E / Z$ configuration was established not only in HeLa cells, but also for numerous other cancer cell lines, including human renal adenocarcinoma cells (ACHN), human 
osteosarcoma cells (HOS, SJSA-1 and drug resistant U2R), and human liver cancer cells (HepG2). As shown in Figure 8b-f, all of them displayed striking toxicities upon incubation with Z-TPAN-POH but good biocompatibilities with the $E$ isomer, suggesting a general trend for distinct toxicities of E/Z-TPAN-POH to cancer cells. The apparent cell morphological changes that ubiquitously happened in all the cell lines after incubation the $Z$ isomer further verified the cell death fates.

To investigate the origin of the difference in toxicity, the enzymatic hydrolytic products, E/Z-TPAN-OH, were first tested. However, no obvious differences in the toxicity could be observed in both $E / Z$ isomers after incubation with HeLa cells. The cellular uptake amount was next evaluated. Strong fluorescence signal from the Z-TPAN-OH fed HeLa cells appeared in almost every cell unit. However, to $E-\mathrm{TPAN}-\mathrm{OH}$, negligible signal could be observed even after irradiation under the mercury lamp for $10 \mathrm{~min}$. The distinctive Raman peak at $2220 \mathrm{~cm}^{-1}$ from the cells incubated with Z-TPAN-POH which could be assigned to the nitrile bond $(\mathrm{C} \equiv$ $\mathrm{N})$ displayed much stronger intensity than the ones incubated with E-TPAN-POH. Further, the HPLC elution profile of the content extracted from the cells incubated with Z-TPAN-POH showed much higher concentration of Z-TPAN-POH precursor and Z-TPAN-OH hydrolysis product in the cells, demonstrating the much faster rate in uptake of the $Z$ isomer by cancer cells when compared to the $E$ isomer. Accompanied by its much slower rate in enzymatic hydrolysis, the massive net accumulation of $\mathrm{Z}-\mathrm{TPAN}-\mathrm{OH}$ in the cancer cells reasonably led to the severe toxicity. This discrepancy in cell viability induced by the stereoisomers are summarized, in which the toxicity from Z-TPAN-POH was attributed to its faster internalization and slower conversion by cancer cells. Simultaneously, the discriminative brightness in fluorescence signal will be contributive for discrimination of the stereoisomers and self-portrait of the toxicity from each isomer, providing a potential tool for the isomeric structure determination, drug screening and therapeutic evaluations.

\section{CONCLUSIONS}

In sum, a series of stereoisomers with nuance in molecular structures while displaying huge disparities in luminescence and biomedical properties were synthesized and separated. The $E / Z$ isomers could be controllably transformed between each other and had been successfully applied in photo-switchable bioimaging by employing the process of photoisomerization. Attributed to their differences in aggregation patterns and enzymatic responsiveness, huge difference in toxicities from the $E / Z$ isomers to numerous types of cancer cells were discovered. The distinctive luminescence properties could serve as an imaging tool to discriminate the $E / Z$ 
configurations and monitor their biomedical activities, promising for visualizable drug screening and pharmaceutical evaluation.

\section{Corresponding Author}

*B.Z.T: tangbenz@ust.hk

\section{Notes}

The authors declare no competing financial interest.

\section{ACKNOWLEDGMENTS}

The authors acknowledge funding to B.Z.T. from the Research Grants Council of Hong Kong (C6009-17G), the Innovation and Technology Commission (ITC-CNERC14SC01), the National Natural Science Foundation of China (21788102), the Natural Science Foundation of Guangdong Province (2019B030301003), and the National Key Research and Development program of China (2018YFE0190200).

\section{REFERENCES}

(1) Boëdec, A.; Sicard, H.; Dessolin, J.; Herbette, G.; Ingoure, S.; Raymond, C.; Belmant, C.; Kraus, J.-L. Synthesis and Biological Activity of Phosphonate Analogues and Geometric Isomers of the Highly Potent Phosphoantigen (E)-1-hydroxy-2-methylbut-2-enyl 4-diphosphate. J. Med. Chem. 2008, 51, 1747-1754.

(2) Chhabra, N.; Aseri, M. L.; Padmanabhan, D. A Review of Drug Isomerism and Its Significance. Int. J. Appl. Basic Med. Res. 2013, 3, 16-18.

(3) Williams, M.; Lennard, M.; Martin, I.; Tucker, G. Interindividual Variation in the Isomerization of 4-hydroxytamoxifen by Human Liver Microsomes: Involvement of Cytochromes P450. Carcinogenesis 1994, 15, 2733-2738.

(4) Katzenellenbogen, B. S.; Norman, M. J.; Eckert, R. L.; Peltz, S. W.; Mangel, W. F. Bioactivities, Estrogen Receptor Interactions, and Plasminogen Activator-Inducing Activities of Tamoxifen and Hydroxytamoxifen Isomers in MCF-7 Human Breast Cancer Cells. Cancer Res. 1984, 44, 112-119.

(5) Wang, D.; Lippard, S. J. Cellular Processing of Platinum Anticancer Drugs. Nat. Rev. Drug Discov. 2005, 4, 307-320.

(6) Stordal, B.; Pavlakis, N.; Davey, R. A Systematic Review of Platinum and Taxane Resistance from Bench to Clinic: an Inverse Relationship. Cancer Treat. Rev. 2007, 33, 688-703.

(7) Coluccia, M.; Natile, G. Trans-Platinum Complexes in Cancer Therapy. Anti-Cancer Agents Med. Chem. 2007, 7, 111-123.

(8) Liang, J.; Shi, H.; Kwok, R. T. K.; Gao, M.; Yuan, Y.; Zhang, W.; Tang, B. Z.; Liu, B. Distinct Optical and Kinetic Responses from E/Z Isomers of Caspase Probes with Aggregation-Induced Emission Characteristics. J. Mater. Chem. B 2014, 2, 4363-4370.

(9) Xu, L.; Zhu, Z.; Wei, D.; Zhou, X.; Qin, J.; Yang, C. Amino-Modified Tetraphenylethene Derivatives as Nucleic Acid Stain: Relationship between the Structure and Sensitivity. ACS Appl. Mater. Interfaces 2014, 6, 18344-18351.

(10) Duan, X.-F.; Zeng, J.; Lü, J.-W.; Zhang, Z.-B. Insights into the General and Efficient Cross McMurry Reactions Between Ketones. J. Org. Chem. 2006, 71, 9873-9876.

(11) Mei, J.; Leung, N. L. C.; Kwok, R. T. K.; Lam, J. W. Y.; Tang, B. Z. Aggregation-Induced 
Emission: Together We Shine, United We Soar! Chem. Rev. 2015, 115, 11718-11940.

(12) He, X.; Zeng, T.; Li, Z.; Wang, G.; Ma, N. Catalytic Molecular Imaging of MicroRNA in Living Cells by DNA - Programmed Nanoparticle Disassembly. Angew. Chem. Int. Ed. 2016, 55, 30733076.

(13) Fan, W.; Yung, B.; Huang, P.; Chen, X. Nanotechnology for Multimodal Synergistic Cancer Therapy. Chem. Rev. 2017, 117, 13566-13638.

(14) Qin, W.; Yang, Z.; Jiang, Y.; Lam, J. W. Y.; Liang, G.; Kwok, H. S.; Tang, B. Z. Construction of Efficient Deep Blue Aggregation-Induced Emission Luminogen from Triphenylethene for Nondoped Organic Light-Emitting Diodes. Chem. Mater. 2015, 27, 3892-3901.

(15) Chen, L.; Jiang, Y.; Nie, H.; Lu, P.; Sung, H. H.; Williams, I. D.; Kwok, H. S.; Huang, F.; Qin, A.; Zhao, Z. Creation of Bifunctional Materials: Improve Electron - Transporting Ability of Light Emitters Based on AIE - Active 2, 3, 4, 5 - Tetraphenylsiloles. Adv. Funct. Mater. 2014, 24, 3621-3630.

(16) Zhao, Z.; Lam, J. W.; Tang, B. Z. Tetraphenylethene: A Versatile AIE Building Block for the Construction of Efficient Luminescent Materials for Organic Light-Emitting Diodes. J. Mater. Chem. 2012, 22, 23726-23740.

(17) Kwok, R. T. K.; Leung, C. W. T.; Lam, J. W. Y.; Tang, B. Z. Biosensing by Luminogens with Aggregation-Induced Emission Characteristics. Chem. Soc. Rev. 2015, 44, 4228-4238.

(18) Guan, W.; Zhou, W.; Lu, C.; Tang, B. Z. Synthesis and Design of Aggregation - Induced Emission Surfactants: Direct Observation of Micelle Transitions and Microemulsion Droplets. Angew. Chem. Int. Ed. 2015, 54, 15160-15164.

(19) Song, Z.; Kwok, R. T. K.; Ding, D.; Nie, H.; Lam, J. W. Y.; Liu, B.; Tang, B. Z. An AlE-Active Fluorescence Turn-on Bioprobe Mediated by Hydrogen-Bonding Interaction for Highly Sensitive Detection of Hydrogen Peroxide and Glucose. Chem. Commun. 2016, 52, $10076-$ 10079.

(20) Feng, X.; Li, Y.; He, X.; Liu, H.; Zhao, Z.; Kwok, R. T. K.; Elsegood, M. R. J.; Lam, J. W. Y.; Tang, B. Z. A Substitution - Dependent Light - Up Fluorescence Probe for Selectively Detecting Fe3+ lons and Its Cell Imaging Application. Adv. Funct. Mater. 2018, 28, 1802833.

(21) Xiong, L.-H.; He, X.; Zhao, Z.; Kwok, R. T. K.; Xiong, Y.; Gao, P. F.; Yang, F.; Huang, Y.; Sung, H. H.-Y.; Williams, I. D.; Lam, J. W. Y.; Cheng, J.; Zhang, R.; Tang, B. Z. Ultrasensitive Virion Immunoassay Platform with Dual-Modality Based on a Multifunctional Aggregation-Induced Emission Luminogen. ACS Nano 2018, 12, 9549-9557.

(22) He, X.; Xiong, L.-H.; Zhao, Z.; Wang, Z.; Luo, L.; Lam, J. W. Y.; Kwok, R. T. K.; Tang, B. Z. AIEBased Theranostic Systems for Detection and Killing of Pathogens. Theranostics 2019, 9, 32233248.

(23) He, X.; Xiong, L.-H.; Huang, Y.; Zhao, Z.; Wang, Z.; Lam, J. W. Y.; Kwok, R. T. K.; Tang, B. Z. AlE-Based Energy Transfer Systems for Biosensing, Imaging, and Therapeutics. TrAC-Trend. Anal. Chem. 2019, 115743.

(24) He, X.; Zhao, Z.; Xiong, L.-H.; Gao, P. F.; Peng, C.; Li, R. S.; Xiong, Y.; Li, Z.; Sung, H. H.-Y.; Williams, I. D.; Kwok, R. T. K.; Lam, J. W. Y.; Huang, C. Z.; Ma, N.; Tang, B. Z. Redox-Active AlEgen-Derived Plasmonic and Fluorescent Core@ Shell Nanoparticles for Multimodality Bioimaging. J. Am. Chem. Soc. 2018, 140, 6904-6911.

(25) He, X.; Peng, C.; Qiang, S.; Xiong, L.-H.; Zhao, Z.; Wang, Z.; Kwok, R. T.; Lam, J. W.; Ma, N.; Tang, B. Z. Less is More: Silver-AIE Core@ Shell Nanoparticles for Multimodality Cancer Imaging and Synergistic Therapy. Biomaterials 2020, 238, 119834.

(26) Niu, G.; Zheng, X.; Zhao, Z.; Zhang, H.; Wang, J.; He, X.; Chen, Y.; Shi, X.; Ma, C.; Kwok, R. T. K.; Lam, J. W. Y.; Sung, H. H. Y.; Williams, I. D.; Wong, K. S.; Wang, P.; Tang, B. Z. AlE-Active 
Functionalized Acrylonitriles: Structure Tuning by Simple Reaction-Condition Variation, Efficient Red Emission and Two-Photon Bioimaging. J. Am. Chem. Soc. 2019, 141, 15111-15120. (27) He, X.; Yin, F.; Wang, D.; Xiong, L.-H.; Kwok, R. T. K.; Gao, P. F.; Zhao, Z.; Lam, J. W. Y.; Yong, K.-T.; Li, Z.; Tang, B. Z. AIE Featured Inorganic-Organic Core@ Shell Nanoparticle for HighEfficiency SiRNA Delivery and Real-Time Monitoring. Nano Lett. 2019, 19, 2272-2279.

(28) Yuan, Y.; Liu, B. Visualization of Drug Delivery Processes Using AIEgens. Chem. Sci. 2017, 8, 2537-2546.

(29) Ji, X.; Shi, B.; Wang, H.; Xia, D.; Jie, K.; Wu, Z. L.; Huang, F. Supramolecular Construction of Multifluorescent Gels: Interfacial Assembly of Discrete Fluorescent Gels through Multiple Hydrogen Bonding. Adv. Mater. 2015, 27, 8062-8066.

(30) Song, N.; Chen, D.-X.; Qiu, Y.-C.; Yang, X.-Y.; Xu, B.; Tian, W.; Yang, Y.-W. StimuliResponsive Blue Fluorescent Supramolecular Polymers Based on a Pillar [5] arene Tetramer. Chem. Commun. 2014, 50, 8231-8234.

(31) Jiang, B.-P.; Guo, D.-S.; Liu, Y.-C.; Wang, K.-P.; Liu, Y. Photomodulated Fluorescence of Supramolecular Assemblies of Sulfonatocalixarenes and Tetraphenylethene. ACS Nano 2014, 8, 1609-1618.

(32) He, X.; Yang, Y.; Guo, Y.; Lu, S.; Du, Y.; Li, J.-J.; Zhang, X.; Leung, N. L.; Zhao, Z.; Niu, G.; Yang, S. W., Zhi; Kowk, R. T. K. L., Jacky W. Y. Xie, Guoming ; Tang, B. Z. Phage-Guided Targeting, Discriminative Imaging, and Synergistic Killing of Bacteria by AIE Bioconjugates. J. Am. Chem. Soc. 2020, 142, 3959-3969.

(33) Mao, D.; Wu, W.; Ji, S.; Chen, C.; Hu, F.; Kong, D.; Ding, D.; Liu, B. ChemiluminescenceGuided Cancer Therapy Using a Chemiexcited Photosensitizer. Chem 2017, 3, 991-1007.

(34) Zhou, C.; Larock, R. C. Regio-and Stereoselective Route to Tetrasubstituted Olefins by the Palladium-Catalyzed Three-Component Coupling of Aryl Iodides, Internal Alkynes, and Arylboronic Acids. J. Org. Chem. 2005, 70, 3765-3777.

(35) Tseng, N.-W.; Liu, J.; Ng, J. C. Y.; Lam, J. W. Y.; Sung, H. H. Y.; Williams, I. D.; Tang, B. Z. Deciphering Mechanism of Aggregation-Induced Emission (AIE): Is E-Z Isomerisation Involved in an AIE Process? Chem. Sci. 2012, 3, 493-497.

(36) Qiu, Z.; Han, T.; Kwok, R. T. K.; Lam, J. W. Y.; Tang, B. Z. Polyarylcyanation of Diyne: A OnePot Three-Component Convenient Route for In Situ Generation of Polymers with AIE Characteristics. Macromolecules 2016, 49, 8888-8898.

(37) Wang, J.; Mei, J.; Hu, R.; Sun, J. Z.; Qin, A.; Tang, B. Z. Click Synthesis, Aggregation-Induced Emission, E/Z Isomerization, Self-Organization, and Multiple Chromisms of Pure Stereoisomers of a Tetraphenylethene-Cored Luminogen. J. Am. Chem. Soc. 2012, 134, 99569966.

(38) Peng, H.-Q.; Zheng, X.; Han, T.; Kwok, R. T. K.; Lam, J. W. Y.; Huang, X.; Tang, B. Z. Dramatic Differences in Aggregation-Induced Emission and Supramolecular Polymerizability of Tetraphenylethene-Based Stereoisomers. J. Am. Chem. Soc. 2017, 139, 10150-10156.

(39) Hong, Y.; Feng, C.; Yu, Y.; Liu, J.; Lam, J. W. Y.; Luo, K. Q.; Tang, B. Z. Quantitation, Visualization, and Monitoring of Conformational Transitions of Human Serum Albumin by a Tetraphenylethene Derivative with Aggregation-Induced Emission Characteristics. Anal. Chem. 2010, 82, 7035-7043.

(40) Leung, C. W. T.; Hong, Y.; Chen, S.; Zhao, E.; Lam, J. W. Y.; Tang, B. Z. A Photostable AIE Luminogen for Specific Mitochondrial Imaging and Tracking. J. Am. Chem. Soc. 2012, 135, 6265.

(41) Zhang, C.-J.; Hu, Q.; Feng, G.; Zhang, R.; Yuan, Y.; Lu, X.; Liu, B. Image-Guided Combination Chemotherapy and Photodynamic Therapy Using a Mitochondria-Targeted Molecular Probe 
with Aggregation-Induced Emission Characteristics. Chem. Sci. 2015, 6, 4580-4586.

(42) Garg, K.; Ganapathi, E.; Rajakannu, P.; Ravikanth, M. Stereochemical Modulation of Emission Behaviour in E/Z Isomers of Diphenyldipyrroethene from Aggregation Induced Emission to Crystallization Induced Emission. Phys. Chem. Chem. Phys. 2015, 17, 19465-19473. (43) Yang, Z.; Qin, W.; Leung, N. L. C.; Arseneault, M.; Lam, J. W. Y.; Liang, G.; Sung, H. H. Y.; Williams, I. D.; Tang, B. Z. A Mechanistic Study of AIE Processes of TPE luminogens: Intramolecular Rotation vs. Configurational Isomerization. J. Mater. Chem. C 2016, 4, 99-107. (44) Hu, R.; Lam, J. W. Y.; Liu, J.; Sung, H. H. Y.; Williams, I. D.; Yue, Z.; Wong, K. S.; Yuen, M. M.; Tang, B. Z. Hyperbranched Conjugated Poly (Tetraphenylethene): Synthesis, AggregationInduced Emission, Fluorescent Photopatterning, Optical Limiting and Explosive Detection. Polym. Chem. 2012, 3, 1481-1489.

(45) Zhang, C. J.; Feng, G.; Xu, S.; Zhu, Z.; Lu, X.; Wu, J.; Liu, B. Structure - Dependent cis/trans Isomerization of Tetraphenylethene Derivatives: Consequences for Aggregation - Induced Emission. Angew. Chem. Int. Ed. 2016, 55, 6192-6196.

(46) Ren, Y.; Xie, S.; Svensson Grape, E.; Inge, A. K.; Ramstrom, O. Multistimuli-Responsive Enaminitrile Molecular Switches Displaying $\mathrm{H}^{+}$-Induced Aggregate Emission, Metal IonInduced Turn-On Fluorescence, and Organogelation Properties. J. Am. Chem. Soc. 2018, 140, 13640-13643.

(47) Fang, X.; Zhang, Y.-M.; Chang, K.; Liu, Z.; Su, X.; Chen, H.; Zhang, S. X.-A.; Liu, Y.; Wu, C. Facile Synthesis, Macroscopic Separation, E/Z Isomerization, and Distinct AIE Properties of Pure Stereoisomers of an Oxetane-Substituted Tetraphenylethene Luminogen. Chem. Mater. 2016, 28, 6628-6636.

(48) Wei, P.; Zhang, J.-X.; Zhao, Z.; Chen, Y.; He, X.; Chen, M.; Gong, J.; Sung, H. H.-Y.; Williams, I. D.; Lam, J. W. Multiple yet Controllable Photoswitching in a Single AIEgen System. J. Am. Chem. Soc. 2018, 140, 1966-1975.

(49) Yu, T.; Ou, D.; Yang, Z.; Huang, Q.; Mao, Z.; Chen, J.; Zhang, Y.; Liu, S.; Xu, J.; Bryce, M. R. The HOF Structures of Nitrotetraphenylethene Derivatives Provide New Insights into the Nature of AIE and a Way to Design Mechanoluminescent Materials. Chem. Sci. 2017, 8, 11631168.

(50) Millán, J. L.; Fishman, W. H.; Stinson, R. Biology of Human Alkaline Phosphatases with Special Reference to Cancer. Crit. Rev. Clin. Lab. Sci. 1995, 32, 1-39.

(51) Jin, C.; He, J.; Zou, J.; Xuan, W.; Fu, T.; Wang, R.; Tan, W. Phosphorylated Lipid-Conjugated Oligonucleotide Selectively Anchors on Cell Membranes with High Alkaline Phosphatase Expression. Nat. Commun. 2019, 10, 1-7.

(52) Liu, H. W.; Li, K.; Hu, X. X.; Zhu, L.; Rong, Q.; Liu, Y.; Zhang, X. B.; Hasserodt, J.; Qu, F. L.; Tan, W. In Situ Localization of Enzyme Activity in Live Cells by a Molecular Probe Releasing a Precipitating Fluorochrome. Angew. Chem. Int. Ed. 2017, 56, 11788-11792.

(53) Zhao, Y.; Zhang, X.; Li, Z.; Huo, S.; Zhang, K.; Gao, J.; Wang, H.; Liang, X. J. Spatiotemporally Controllable Peptide - Based Nanoassembly in Single Living Cells for a Biological Self - Portrait. Adv. Mater. 2017, 29, 1601128.

(54) Benham, F. J.; Harris, H. Human Cell Lines Expressing Intestinal Alkaline Phosphatase. Proc. Natl. Acad. Sci. USA 1979, 76, 4016-4019.

(55) Lange, P. H.; Millan, J. L.; Stigbrand, T.; Vessella, R. L.; Ruoslahti, E.; Fishman, W. H. Placental Alkaline Phosphatase as a Tumor Marker for Seminoma. Cancer Res. 1982, 42, 32443247. 


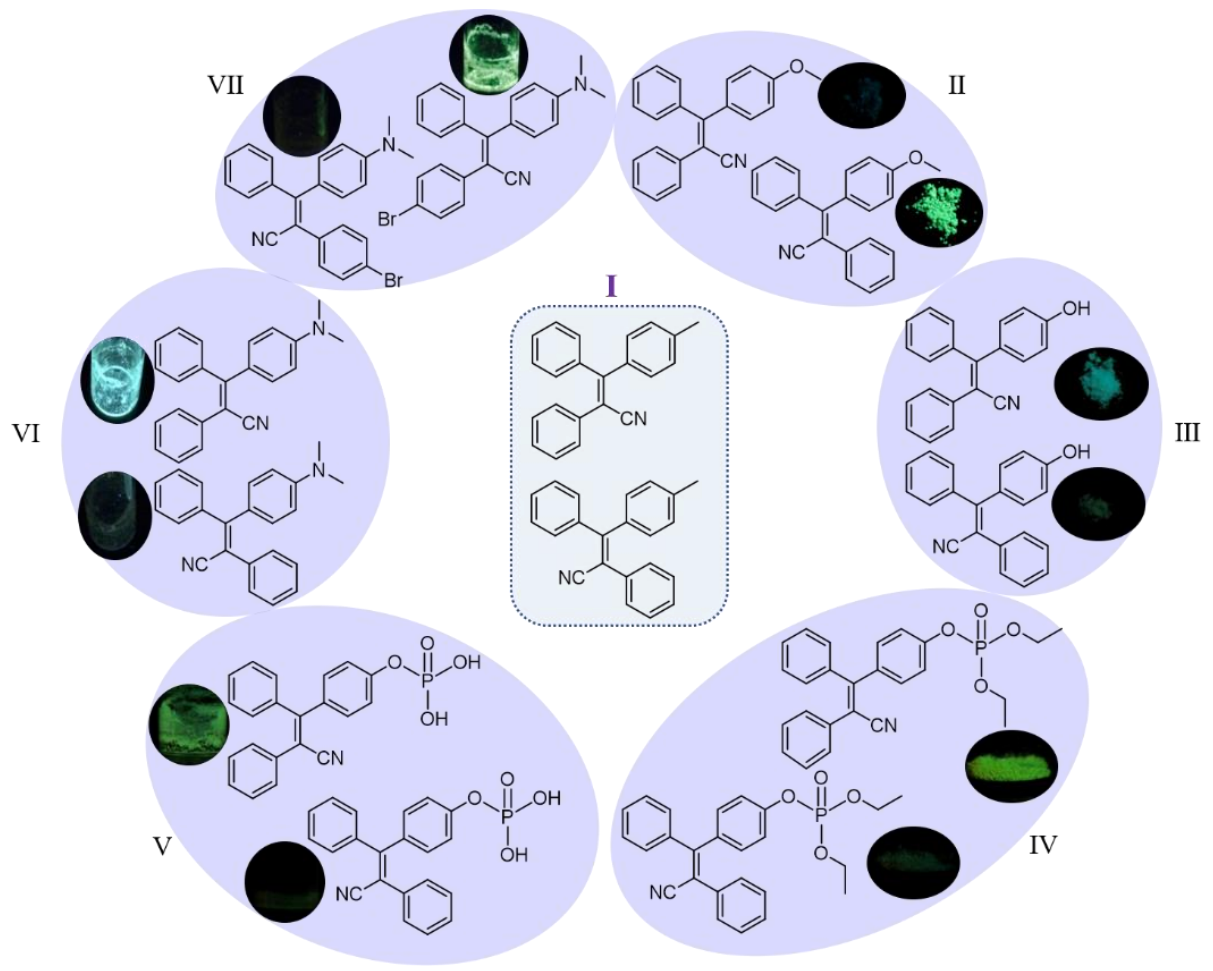

Scheme 1. Molecular structures of a series of AIE stereoisomers with their solid-state fluorescent pictures under $365 \mathrm{~nm}$ UV irradiation (I: E/Z-TPAN-M; II: E/Z -TPAN-OM; III: E/Z -TPAN-OH; IV: E/Z -TPAN-POE; V: E/Z -TPAN-POH; VI: E/Z -TPAN-NM; VII: E/Z -TPAN-NMBr).
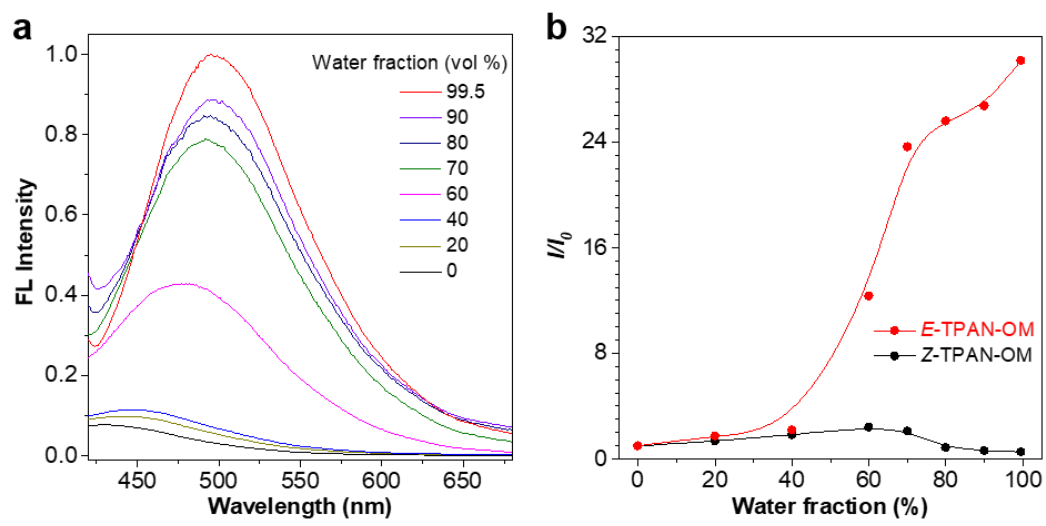

Figure 1. AIE property characterization. (a) fluorescence spectra of $E$-TPAN-OM in mixture of DMSO and water with varying fraction of water, respectively. (b) The evolution of intensity in the fluorescence emission peak of E/Z-TPAN-OM with varying fraction of water, respectively. 

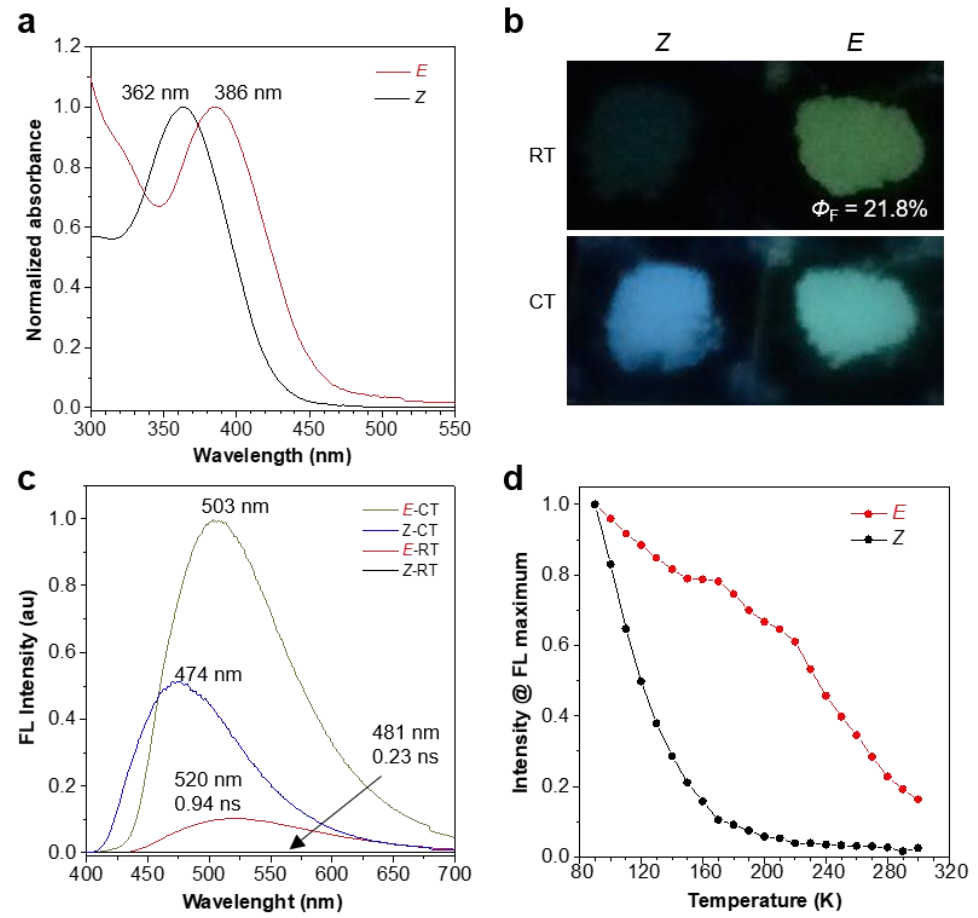

Figure 2. Optical property characterization of E/Z-TPAN-OM. (a) Absorption spectra of solid E/ZTPAN-OM, respectively, with absorption maximum labelled. (b) Fluorescent pictures of E/Z-TPANOM powder at RT and CT under UV light, respectively. The absolute quantum yield $\left(\Phi_{\mathrm{F}}\right)$ was labelled for $E$-TPAN-OM. (c) Fluorescent spectra of solid E/Z-TPAN-OM under room temperature (RT) or cryogenic temperature (CT, $77 \mathrm{~K})$, respectively. The decay lifetime $(\tau)$, emission wavelength $(\lambda)$ were labelled. (d) Temperature dependent fluorescence emission intensity of E/Z-TPAN-OM, respectively.
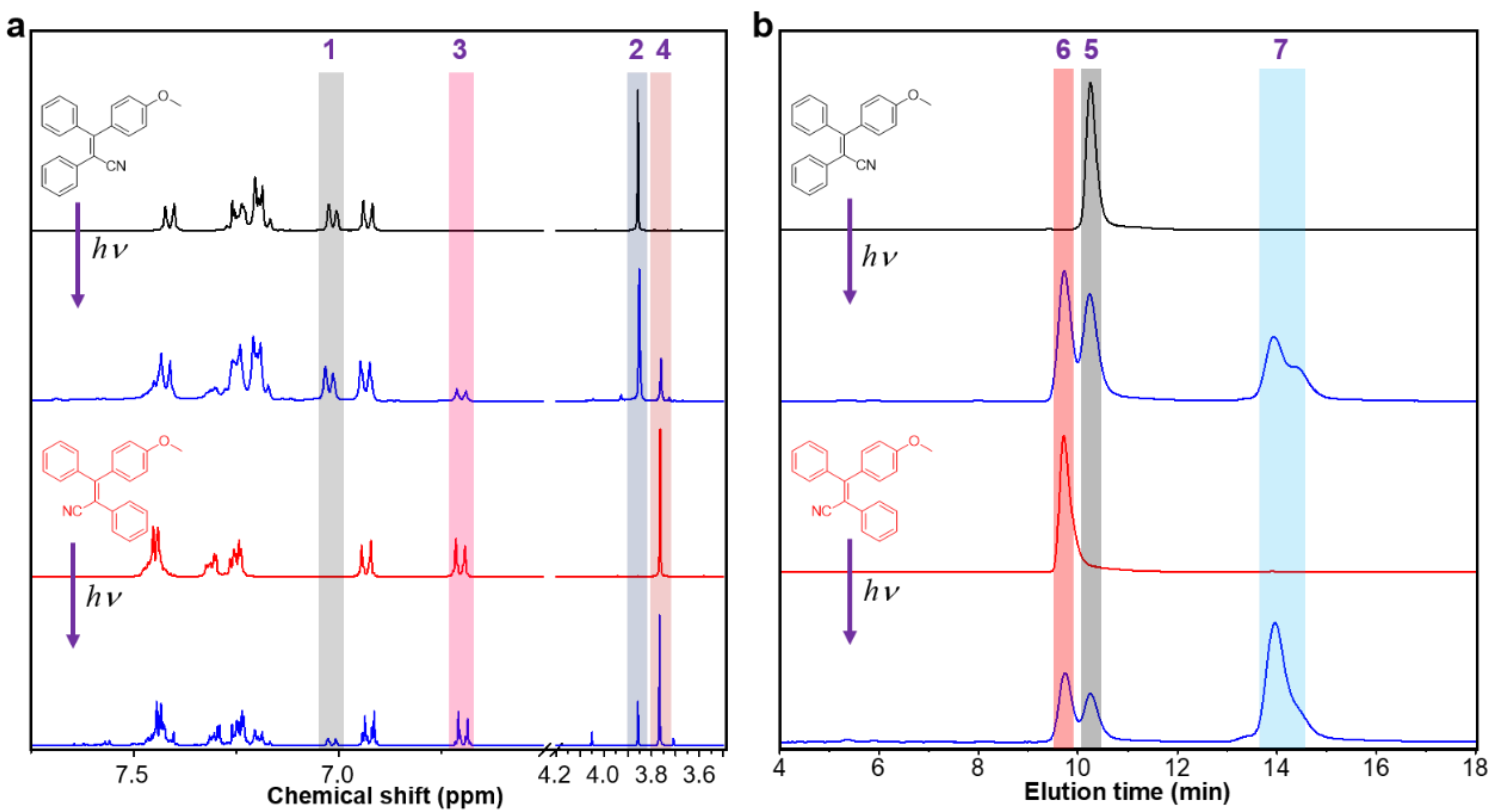

Figure 3. Isomer transformation tests of E/Z-TPAN-OM under $365 \mathrm{~nm}$ UV irradiation for $150 \mathrm{~min}$. (a) Monitored by ${ }^{1} \mathrm{H} \mathrm{NMR}$ in $\mathrm{CDCl}_{3}$. The proton resonances in the aromatic ring in zone-1and zone-3 belonged to $Z$ and $E$-TPAN-OM, respectively. And the proton resonances in methyl group in zone- 2 and zone-3 were assigned to $Z$ and $E$-TPAN-OM, respectively. (b) Monitored by HPLC at $280 \mathrm{~nm}$. The elution peaks in zone- 5 and zone- 6 belonged to $Z$ and $E$-TPAN-OM, respectively. The elution peak in zone-7 was derived from their photocyclization products. 


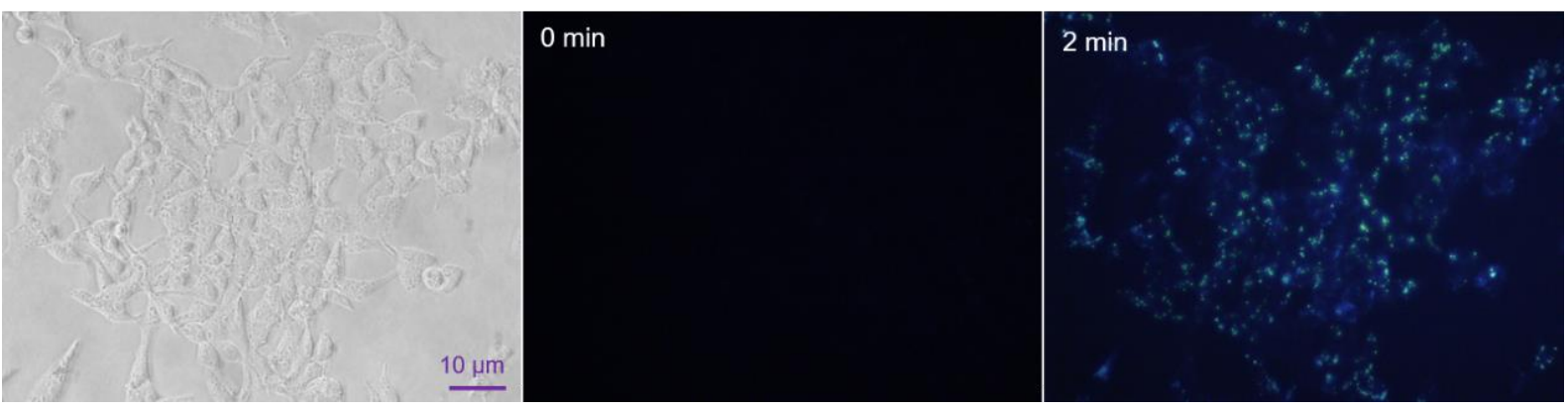

Figure 4. Photo-switchable cell imaging. HeLa cells incubated with Z-TPAN-OM in bright-field, fluorescence imaging at $0 \mathrm{~min}$, and fluorescence imaging after irradiation under a mercury lamp for 2 min, respectively.
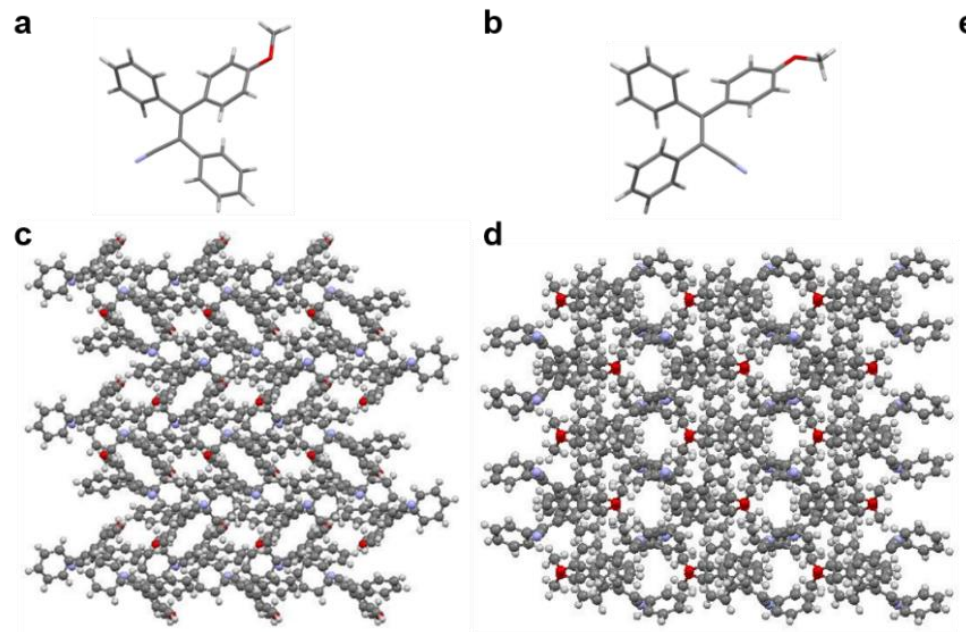

\begin{tabular}{cccc}
\hline$T(\mathrm{~K})$ & $V_{\mathrm{V}, \mathrm{E}}\left(\AA^{3}\right)$ & $V_{\mathrm{V}, \mathrm{Z}}\left(\AA^{3}\right)$ & $V_{\mathrm{V}, \mathrm{Z}} / V_{\mathrm{V}, \mathrm{E}}$ \\
\hline 100 & 0.356 & 2.756 & 7.74 \\
140 & 0.326 & 2.859 & 8.77 \\
160 & 0.343 & 3.111 & 9.07 \\
180 & 0.393 & 3.163 & 8.05 \\
200 & 0.393 & 3.307 & 8.41 \\
220 & 0.437 & 3.532 & 8.08 \\
240 & 0.504 & 3.644 & 7.23 \\
270 & 0.621 & 3.979 & 6.41 \\
293 & 0.708 & 4.124 & 5.82 \\
\hline
\end{tabular}

Figure 5. (a, b) Crystal structure of E/Z-TPAN-OM under 293 K, respectively. (c, d) Packing pattern of E/Z-TPAN-OM under $293 \mathrm{~K}$, respectively. (e) Evolution of volume of void $\left(V_{\mathrm{V}}\right)$ in crystal packing of E/Z-TPAN-OM by measuring the space outside the molecular surface with $1.0 \AA$ distance and their calculated ratios under varying temperatures. 

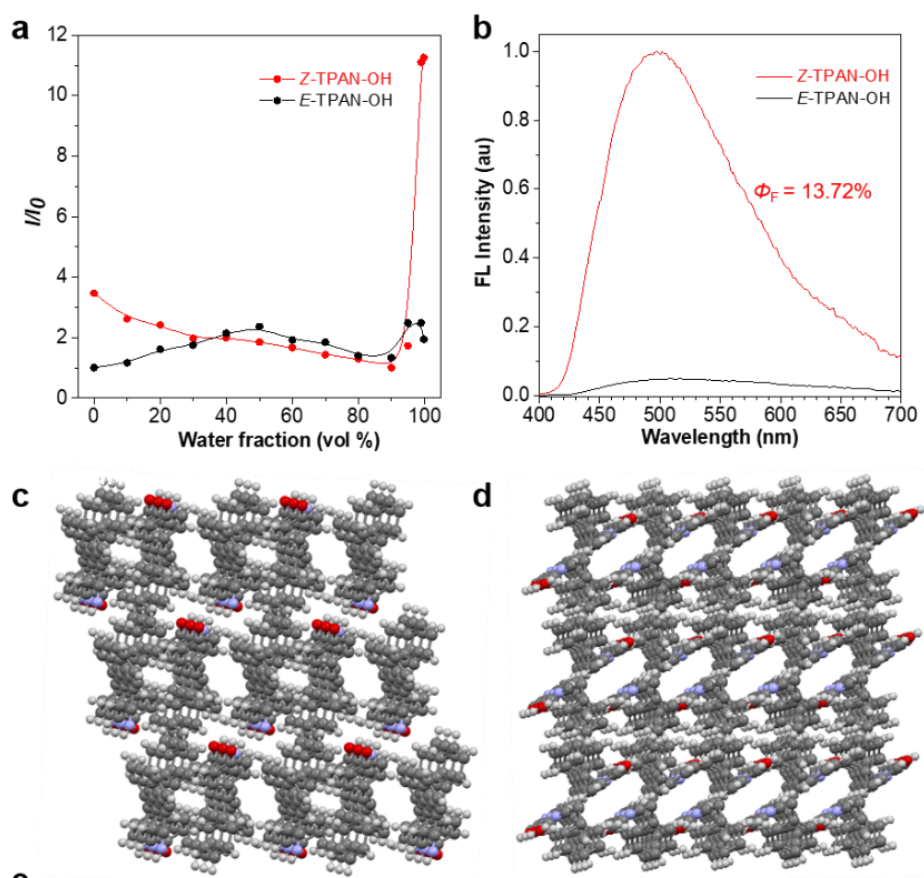

\begin{tabular}{cccc}
\hline$T(\mathrm{~K})$ & $V_{\mathrm{V}, \mathrm{Z}}\left(\AA^{3}\right)$ & $V_{\mathrm{V}, \mathrm{E}}\left(\AA^{3}\right)$ & $V_{\mathrm{V}, \mathrm{E}} / V_{\mathrm{V}, \mathrm{Z}}$ \\
\hline 100 & 0.243 & 5.394 & 22.20 \\
150 & 0.315 & 5.838 & 18.53 \\
180 & 0.347 & 6.129 & 17.66 \\
200 & 0.333 & 6.383 & 19.17 \\
220 & 0.349 & 6.737 & 19.30 \\
250 & 0.397 & 6.972 & 17.56 \\
\hline
\end{tabular}

Figure 6. (a) AIE curve of $\mathrm{Z} / E-\mathrm{TPAN}-\mathrm{OH}$ in the mixture of $\mathrm{DMSO} / \mathrm{H}_{2} \mathrm{O}$. (b) Fluorescence spectra of $Z / E$-TPAN-OH crystals with absolute quantum yield $\left(\Phi_{\mathrm{F}}\right)$ was labelled for Z-TPAN-OH under RT. (c) Crystal packing pattern of Z-TPAN-OH under $250 \mathrm{~K}$, (d) (c) Crystal packing pattern of $E$-TPAN-OH under $250 \mathrm{~K}$. (f) Evolution of volume of void $\left(V_{\mathrm{V}}\right)$ in crystal packing of Z/E-TPAN-OH by measuring the space outside the molecular surface with $1.0 \AA$ distance and their calculated ratios under varying temperatures.

a

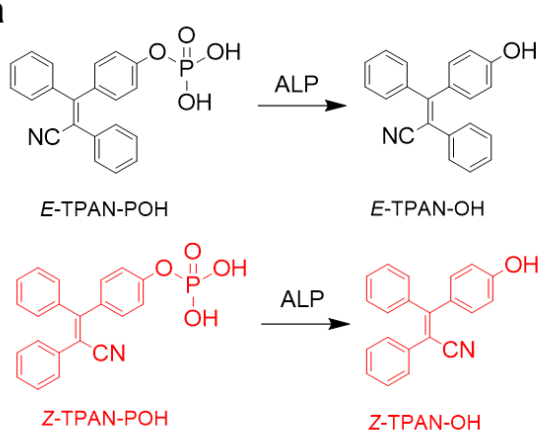

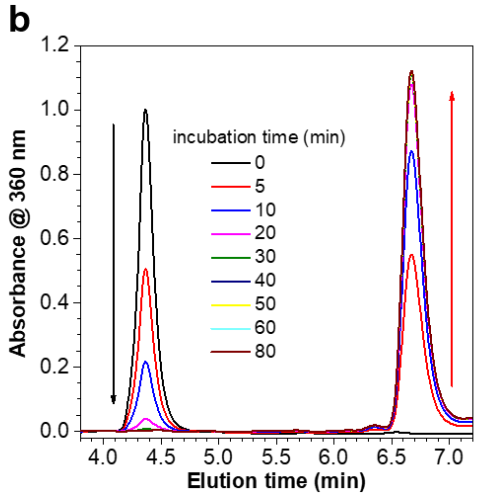

Figure 7. (a) The enzymatic reaction from E/Z-TPAN-POH to E/Z-TPAN-OH. (b) ALP catalyze hydrolysis of Z-TPAN-POH monitored by HPLC. (c) The calculated hydrolysis conversion rates of $E / Z$ TPAN-POH. The concentration of E/Z-TPAN-POH was $100 \mu \mathrm{M}$, ALP was $1.05 \mathrm{nM}$. 
a
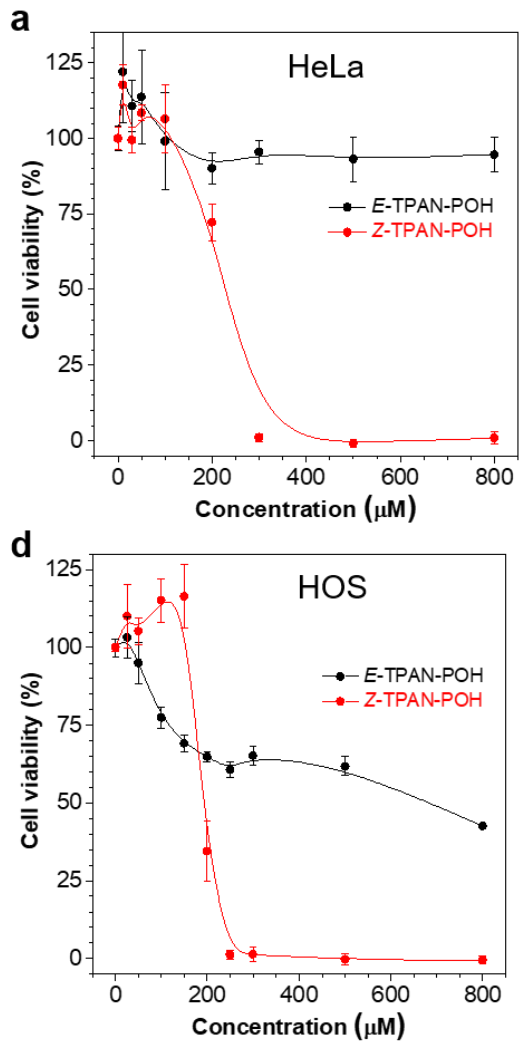

b
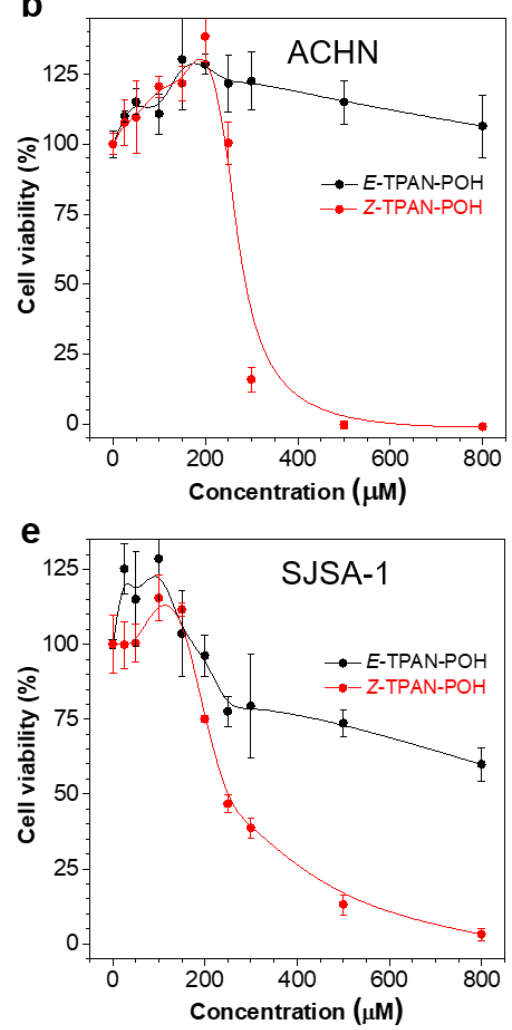

C
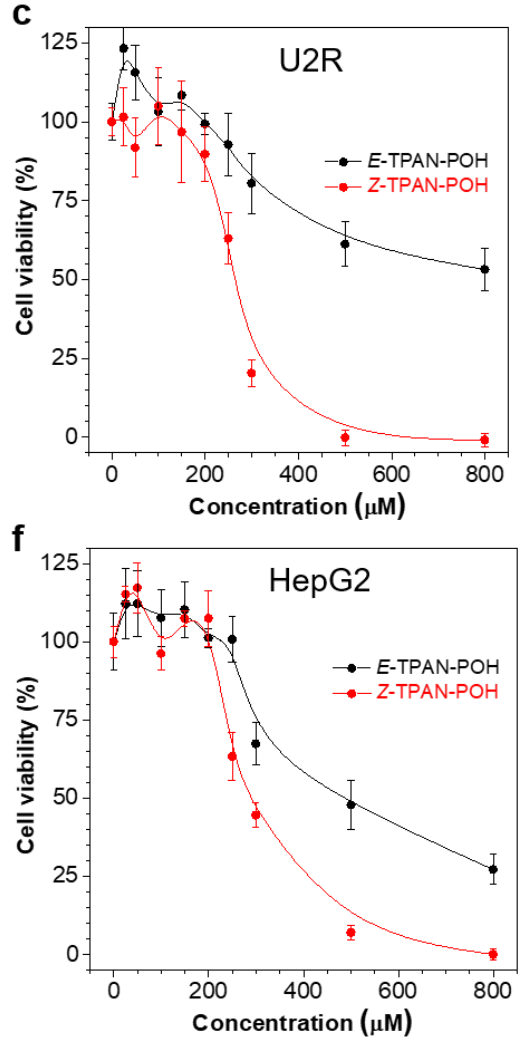

Figure 8. Cellular viability tests of E/Z-TPAN-POH in various types of cancer cell lines. Varying concentration of E/Z-TPAN-POH incubated with (a) HeLa cells for 2 hours, (b) ACHN cells for 12 hours, (c) U2R cells for 6 hours, (d) HOS cells for 12 hours, (e) SJSA-1 cells for 6 hours, and (f) HepG2 cells for 2 hours, respectively. 